\title{
Microelectrode array systems for their use in single nanowire-based gas sensor platforms
}

\author{
Ondřej Chmela, ${ }^{*}$ Jakub Sadílek, \\ Stella Vallejos, ${ }^{* *}$ Jaromír Hubálek $^{*, * *}$
}

\begin{abstract}
Microelectrode array systems for their use in single-nanowire-based gas sensor platforms are developed. The topology of the system is designed with the aim of determining the optimal conditions and the factors involved in the selective integration of gas sensitive semiconducting metal oxide nanowires via dielectrophoresis method. Thus various electrode geometries with electrode gaps between 2 and $10 \mu \mathrm{m}$ and electrode tip-end shapes are investigated employing tungsten oxide nanowires synthetized via aerosol-assisted chemical vapor deposition. Results obtained from SEM, optical microscopy and electrical tests demonstrate that the integration and electrical contact of single nanowires across the electrodes is achieved in the systems with electrode gaps below $3 \mu \mathrm{m}$. These results are discussed and further improvements in the design of these systems are suggested.
\end{abstract}

K e y w o r d s: nanowires, microelectrode arrays, dielectrophoresis, gas sensors

\section{Introduction}

In recent years, nanomaterial-based chemical sensors have shown great potential for their application in areas such as medicine, industry, environment, agriculture or security, to cite a few. The use of nanostructures (eg nanowires, nanotubes, nanorods or nanobelts) has shown to play significant role in the functionality of these devices, not only due to their high surface area to volume ratio, but also due to the formation of specific crystal facets and in turn specific electronic structure at their surface, which have shown to provide enhanced chemical and physical properties to the material. [1] Particularly in gas sensors, nanostructures have demonstrated to improve the functionality of the sensor providing better sensitivity, selectivity and stability. [2] [3] Nanostructures have also allowed for miniaturization of the sensor device, and in this line (single) nanowire-based gas sensors have shown potential for reducing fabrication cost, material and power consumption. [4]

Generally, two methods are used to fabricate nanowirebased sensors. The first based on top-down processes, which often increase the fabrication cost, and the second based on the re- deposition of nanowires, previously synthesised by bottom-up processes either using vapourphase or liquid-phase routes. [5] Present work focuses on the second approach and makes use of a bottomup process via aerosol-assisted chemical vapor deposition (AACVD) to synthesize nanowires and the dielectrophoresis method to integrate and interconnect the nanowires into microelectrode arrays designed to operate in resistive mode. AACVD is a variant of traditional
CVD in which a precursor solution is transported to the substrate in the form of aerosol. This method has shown to produce nanostructured metal oxides $\left(e g \mathrm{WO}_{3}\right)[6]$ by controlling the degree of homogenous and heterogeneous gas-phase reaction via different process conditions such as the deposition temperature, carrier solvents and concentration of reactive species. $[7,8]$

The dielectrophoresis technique consists in applying a controlled volume of nanowires suspended on any liquid (eg water, isopropyl alcohol) to enable the nanowires to reach the microelectrode system with applied electrical potential. This technique uses an alternate electric field to induce nanowire movement effect on the substrate surface and thus provide an accurate control of the alignment and contact of nanowires across a couple of electrodes. $[9,10]$

Here, we develop a chip based on microelectrode systems for the fabrication of single- nanowire-based sensor arrays using dielectrophoresis method, and discuss the favorable and the unfavorable factors of this design for the optimal integration of tungsten oxide nanowires synthetized via AACVD.

\section{Fabrication process}

\section{Microelectrode system}

Several microelectrode systems $(1 \times 1 \mathrm{~cm}$, Fig. 1) were fabricated in a 4 -inch silicon wafer $(525 \mu \mathrm{m}$ thick, Microchemical, fused silica, arsenic doped), electrically isolated by a thermally grown silicon dioxide layer $(500$ $\mathrm{nm}$ thick). Gold (100 nm thick) was used as conductive

* Brno University of Technology, Department of Microelectronics, Technická 3058/10, 61600 Brno, Czech Republic, ** CEITEC - Central European Institute of Technology, Brno University of Technology, Purky]vnova 123, 61200 Brno, Czech Republic, vargas@feec.vutbr.cz 


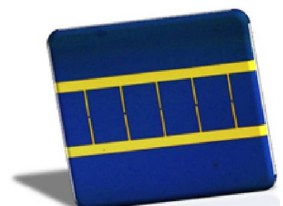

(a)

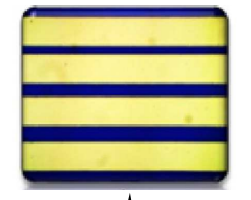

(b) ${ }^{\wedge}$
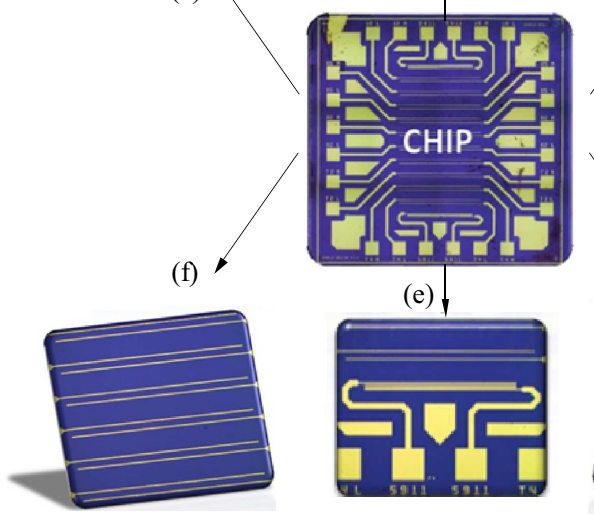

(e) ,

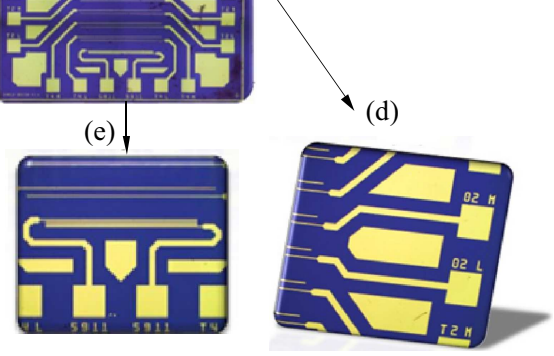

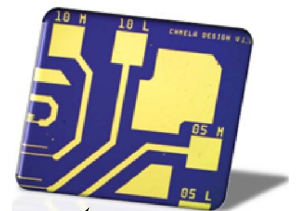

(c)

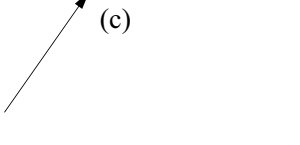

Fig. 1. Chip topology; (a) - microelectrode array, (b, e) - test contacts, (c, d, e) - electrode pad contacts, (c, d) - hydrophobic stop layer, and (f) - parallel-connected microelectrode array

layer due to its high conductivity and non-corrosive properties that allow for the use of a liquid media during the re-deposition of nanowires on the chip. Titanium (5 $\mathrm{nm}$ thick) was used as adhesive layer, between the conductive gold layer and the silicon oxide dielectric layer. Both layers were sputtered by ion-beam deposition system (Bestec) equipped with RFICP Kaufman ion-beam source (Kaufman \& Robinson - KRI@). The deposition parameters (thickness and rate of deposition) were monitored by Inficon SQM-242 card with a quartz crystal sensor as reported previously [11].

The lithography process was carried out using UV direct write laser system (Heidelberg Instruments, DWL 66fs), and the process conditions of development steps were set according to the specifications provided by the producer of the photoresist (Allresist, AR-N 4340). This process led to a minimal resolution of $1 \mu \mathrm{m}$ approximately. The gold layer was also used as hydrophobic layer to avoid liquid expansion through the whole chip surface and confine the drop containing the suspended nanowires in the area of electrodes during the dielectrophoresis process, thus whole chip was fabricated with only one lithographic step, Fig. 1(c) and 1(d) show the hydrophobic stop layer in the chip. After developing process of photoresist, gold layer was wet-etched (Sigma-Aldrich, Gold etchant - standard). Subsequently, the titanium layer was etched by solution of hydrofluoric acid, nitric acid and deionized water in ratio $1 \mathrm{HF}: 1 \mathrm{HNO}_{3}: 20 \mathrm{H}_{2} \mathrm{O}$. The etching time of the titanium layer with thickness about $5 \mathrm{~nm}$ was dependent on the thickness of native oxide above, as the native oxide turns to be more resistant for etchant than the titanium layer. Thus the time adjusted for this etching step was $30 \mathrm{~s}$. Then the samples were electrically tested on a probe station (Cascade Microtech, M150). After development process, the whole silicon wafer along with the structures was spin- coated with a positive photoresist S1813 (MicropositTM). This layer was used as a protecting layer to avoid scratches and impurities during the cutting step, which was performed using ohmical trimming laser (Aurel Automation, ALS300) to divide the silicon wafer to chip pieces of $1 \times 1 \mathrm{~cm}$. Finally, the silicon wafer was cleaned from resists residual and other organic compounds using PG Remover solution (Microchem). The solution was heated to $80^{\circ} \mathrm{C}$ and introduced into sonication for more than $30 \mathrm{~min}$. To achieve maximum cleaning effect and to prevent the re-depositions of resist residues this process was repeated twice. Then, the silicon wafer was two times rinsed with deionized water and dried with nitrogen.

To integrate several nanowires in one step via dielectrophoresis method, the chip contained 12 test contact arrays, Fig. 1(f) with different specification ie electrode gap and electrode tip-end shapes. As we found the nanowires after sonication have maximum lengths about $12 \mu \mathrm{m}$, we determined to use electrode gaps below this value, with the minimal and maximum electrode gap fabricated about $2 \mu \mathrm{m}$ and $10 \mu \mathrm{m}$, respectively. Thus, each chip consisted of two test structures, each with 4 lines of electrode arrays, shown in Fig. 1(b), 1(e). The electrodes were designed with a constant width but different electrode gaps to find out the optimal gaps for the AACVD deposited nanowires. Then, each array contained 10 electrodes with different spacing between electrodes (50 and $100 \mu \mathrm{m})$, Fig.1(a), Fig 2. Different tip-end shapes eg in the form of round or triangle, shown in Fig. 2, were also designed to study their influence on the dielectrophoresis processes. Each array of electrodes was provided of two contact pad $(100 \times 100 \mu \mathrm{m})$ as shown in Fig.1(c), 1(d), and $1(\mathrm{e})$. 


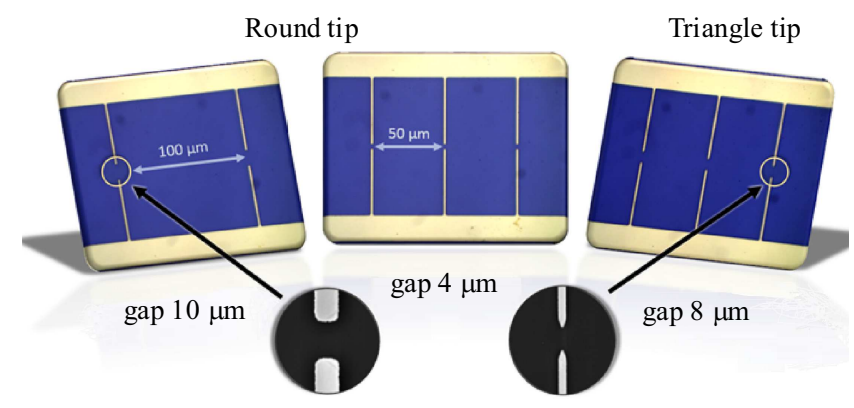

Fig. 2. Detailed view of the array with different electrode gap and contact types

\section{Nanowire synthesis}

Tungsten oxide nanowires were grown from tungsten hexacarbonyl $\left(\mathrm{W}(\mathrm{CO})_{6}, 20 \mathrm{mg}\right.$, Sigma- Aldrich, $\geq 97 \%$ ) on tiny silicon tiles $(1 \times 1 \mathrm{~mm})$ employing the AACVD system described previously [6] (tiny substrates were used to reduce the number of suspended nanowires in the solution after removal, and thus suppress the large number of re-deposited nanowires on the surface during the dielectrophoresis process). Briefly, the conditions to grow tungsten oxide nanowires from $\mathrm{W}(\mathrm{CO}) 6$ via AACVD involved a screening of deposition temperatures, solvents and solution concentrations finding the optimal condition for the growth of nanowires at $390{ }^{\circ} \mathrm{C}$ using methanol $(5 \mathrm{ml}$, Penta, $\geq 99.8 \%$ ). A commercial piezoelectric ultrasonic atomizer was used to generate aerosol from the methanolic solution of $\mathrm{W}(\mathrm{CO})_{6}$, and the aerosol droplets were transported to the heated substrate using a controlled nitrogen gas flow $(200 \mathrm{sccm})$ that took about 70 minutes to transport the entire volume of solution.

\section{Characterization and nanowire integration}

The samples were examined using scanning electron microscopy (SEM; Tescan FE Mira II LMU) equipped with EDX, and the phase of the AACVD deposited films using X-ray diffraction (XRD; Rigaku SmartLab 3 $\mathrm{kW}, \mathrm{Cu} K \alpha$ radiation). To integrate the nanowires with the microelectrode system by dielectrophoresis method, firstly, the as-deposited nanowires were removed from the silicon substrate and suspended in water using sonication (Bandelin SONOREX TM, Digital 10P), and then a contact-angle measurement station (SEO, Phoenix 300) and a probe station provided of a signal generator (Agilent, 33220A, $20 \mathrm{MHz}$ function/arbitrary waveform generator) were employed.

\section{Results and discussion}

The AACVD of $\mathrm{W}(\mathrm{CO})_{6}$ at $390{ }^{\circ} \mathrm{C}$ resulted in the formation of adherent uniform bluish films. The formation of tungsten oxide films from AACVD of $\mathrm{W}(\mathrm{CO})_{6}$ is attributed to the use of oxygen-containing solvent, such as methanol, and the decomposition of $\mathrm{W}(\mathrm{CO})_{6}$ via the loss of the carbonyl group.[12] EDX analysis in different points of the as-deposited films indicated $\mathrm{O} / \mathrm{W}$ ratios of $\approx 2.7$ suggesting the formation of partially reduced tungsten oxide films, and the XRD patterns (Fig. 3) displayed intense diffraction peaks at $23.5 \mathrm{deg} 2 \theta(d=3.77 \AA)$ and $48.2 \operatorname{deg} 2 \theta(d=1.88 \AA)$ associated to a monoclinic phase $\mathrm{WO}_{3}$ (P21/n space group, ICCD card no. 72-0677) with preferred orientation in the [010] direction, consistent with the XRD patterns observed in our previous work. [6] Top and cross sectional SEM images (Fig. 4) of the films revealed the formation of non-aligned nanowire structures with diameters of $50-150 \mathrm{~nm}$ and lengths of $15 \mu \mathrm{m}$, approximately.

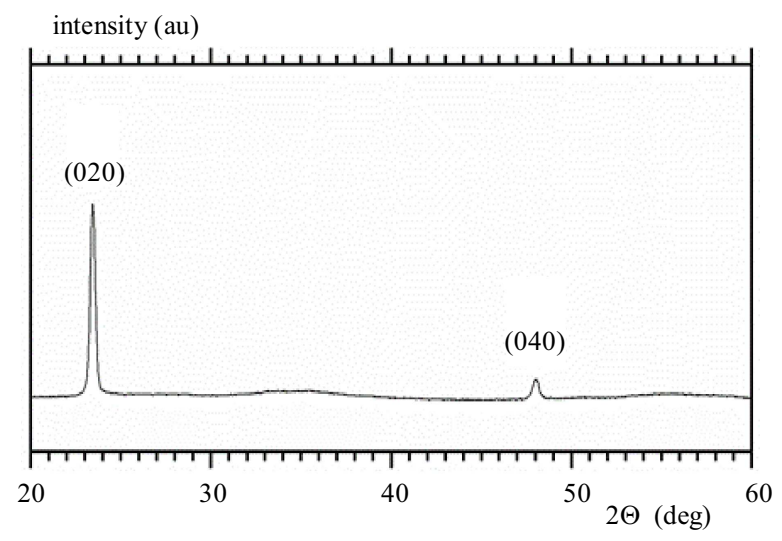

Fig. 3. Typical XRD patterns recorded on the as-deposited tungsten oxide nanowires films deposited via AACVD
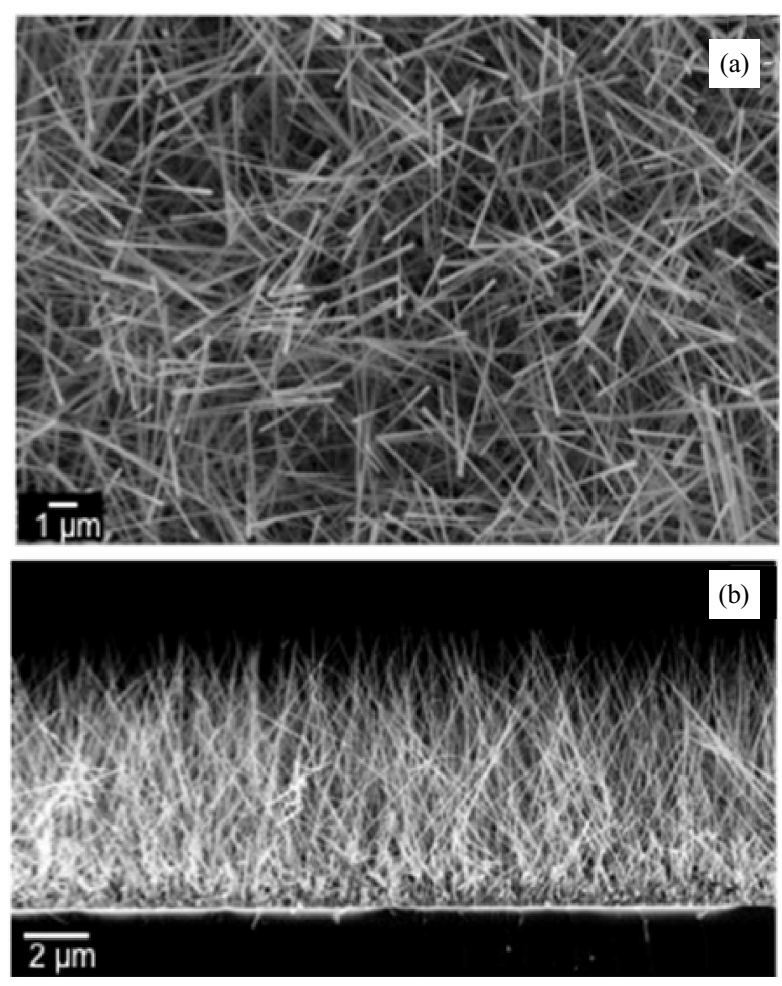

Fig. 4. Top (a) - and sectional, (b) - view of the as-deposited nanowires on silicon tiles using SEM 

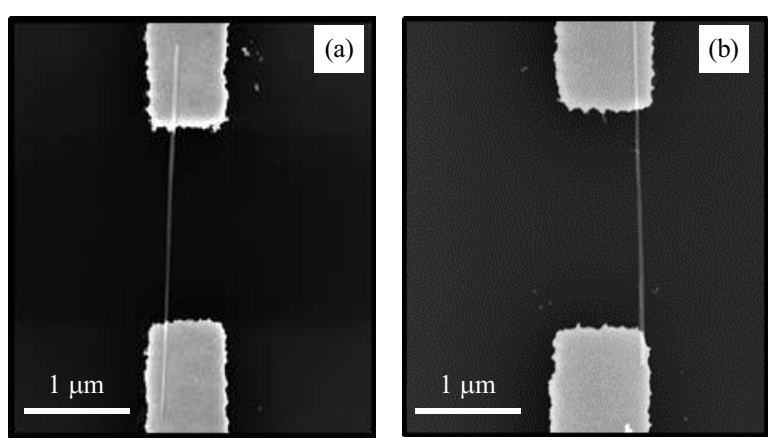

Fig. 5. Typical alignement of nanowires accross two diferent electrodes of the array

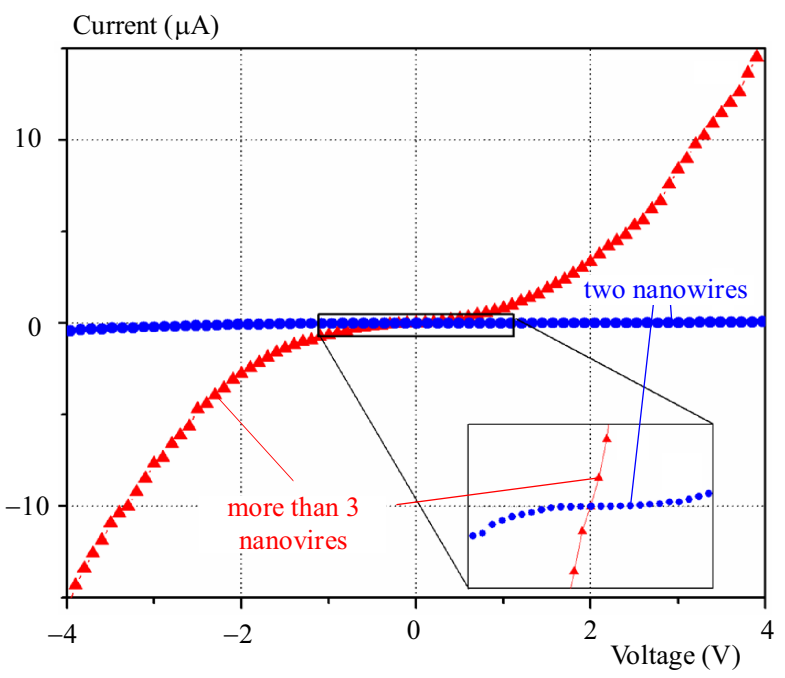

Fig. 6. I-V characteristics tested at room temperature for an array of two nanowires (1) and more than three nanowires (2)

To set up optimal conditions for the dielectrophoresis process, firstly we optimized the removal of the asdeposited nanowires from the substrate by sonication (Bandelin SONOREXTM, Digital 10P), preventing damages of the structures in particular avoiding the shortening of the nanowire length. To this end, we investigated several time periods (1, 3, 5, 10 and 15 seconds) and power intensities for sonication (10, 20 and 100\%) using deionized water. The maximum of sonication (ie 100\%) damaged the nanowires reducing them into small size pieces, below the minimum electrode gap fabricated. Thus, the optimal conditions for this process were found when using a power intensity for sonication of $10 \%$ and $35 \mathrm{kHz}$ during 5 seconds. These conditions allow the separation of nanowires with maximum lengths of $12 \mu \mathrm{m}$ approximately, consistent with the electrode gaps fabricated in our chip. After removal and suspension of the asdeposited nanowires in water, a suitable volume of drops (1 $\mu \mathrm{l}$ approximately) were adjusted and controlled using the contact angle measurement station (we noticed that this small volumes are relevant to control and encourage selective integration of single nanowires across each couple of electrodes). Consequently, the chip containing a drop was connected to the probe station, where an alternating voltage potential was applied. These tests showed that by using short holding voltage time cycles from 10 to 20 seconds with applied voltage of $5 \mathrm{~V}$ and frequency of $7 \mathrm{MHz}$, single nanowires are able to contact across the microelectrode. Fig. 5 displays a typical SEM view of the single nanowires connected across the electrodes after the dielectrophoresis process.

Electrical measurements of a system containing an array of two nanowires (1) and another containing more than three nanowires (2) are shown in Fig. 6. These results indicate an asymmetric and non-linear characteristic for the I-V curves in both systems suggesting that the contact between the nanowire and the electrode has a Schottky nature. In gas sensors the response is defined as the relative changes of electrical resistance before and after exposure to gaseous analysts, and generally two components contribute to these changes: the nanowire channel and the contact resistance between nanowire and the electrode. These contacts may have a Schottky or ohmic nature, and although ohmic contacts tend to be more popular in gas sensors, there is evidence that Schottky contacts are more appropriate for reducing gases enhancing the sensor response, for instance up to four orders of magnitude, as demonstrated previously for carbon monoxide sensors. [13,14]

\section{Conclusions}

A microelectrode array system for the fabrication of single nanowire-based sensors has been developed. This microarray has shown to be suitable for the alignment and electrical connection of single nanowires across the electrodes using a dielectrophoresis process with adjusted bias voltage and frequency to $5 \mathrm{~V}$ and $7 \mathrm{MHz}$, respectively, for 10 to 20 seconds. We found that the electrodes with gaps below $3 \mu \mathrm{m}$ are the most convenient to build these systems as the nanowire length were highly dispersed and the probability to place the longer nanowires across the structure was very low. The influence of the tipend shape on the integration process of nanowires was not evident in this work, most likely due to its microscale features. Further work on the fabrication of electrodes with electrode finger-widths closely related to the diameter of nanowires is in progress.

\section{Acknowledgements}

This work has been supported by the Czech Science Foundation (GAČR) via Grant no. 17-16531S. O. Ch. acknowledges the project FEKT-S-17-3934 (Utilization of novel findings in micro and nanotechnologies for complex electronic circuits and sensor applications). The support of the National Sustainability Program under grant LO1401 and the access to the infrastructures of CEITEC Nano Research (ID LM2015041, MEYS CR, 20162019) and SIX Research Centre are also acknowledged. 


\section{REFERENCES}

[1] N. S. Ramgir, Y. Yang and M. Zacharias, "Nanowire-Based Sensors", Small, vol. 6, pp. 1705-1722, 2010.

[2] D. R. Miller, S. A. Akbar and P. A. Morris, "Nanoscale metal oxide-based heterojunctions for gas sensing: A review", Sensors \& Actuators: B. Chemical, vol. 204, pp. 250-272, 2014.

[3] A. Gurlo, "Nanosensors: towards morphological control of gas sensing activity", $\mathrm{SnO} 2, \mathrm{In} 2 \mathrm{O} 3, \mathrm{ZnO}$ and WO3 case studies.Nanoscale, vol. 3, pp. 154-165, 2011.

[4] H. Huang, B. Liang, Z. Liu, X. Wang, D. Chen and G. Shen, "Metal oxide nanowire transistors", Journal of Materials Chemistry, vol. 22, pp. 13428-13445, 2012.

[5] R. G. Hobbs, N. Petkov and J. D. Holmes, "Semiconductor Nanowire Fabrication by Bottom-Up and Top-Down Paradigms", Chemistry of Materials, vol. 24, pp. 1975-1991, 2012.

[6] S. Vallejos, I. Grácia, O. Chmela, E. Figueras, J. Hubálek and C. Cané, "Chemoresistive micromachined gas sensors based on functionalized metal oxide nanowires: Performance and reliability", Sensors and Actuators B: Chemical, vol. 235, pp. 525-534, 2016.

[7] S. Vallejos, P. Umek and C. Blackman, "Aerosol Assisted Chemical Vapour Deposition Control Parameters for Selective Deposition of Tungsten Oxide Nanostructures", Journal of Nanoscience and Nanotechnology, vol. 11, pp. 8214-8220, 2011.

[8] M. Ling and C. Blackman, "Growth mechanism of planar or nanorod structured tungsten oxide thin films deposited via aerosol assisted chemical vapour deposition (AACVD)", physica status solidi (c), vol. 12, pp. 869-877, 2015.

[9] N. K. R. Palapati, E. Pomerantseva and A. Subramanian, "Single nanowire manipulation within dielectrophoretic force fields the sub-crossover frequency regime", Nanoscale, vol. 7, pp. 3109-3116, 2015.

[10] S. Raychaudhuri, S. A. Dayeh, D. Wang and E. T. Yu, "Precise Semiconductor Nanowire Placement Through Dielectrophoresis", Nano Letters, vol. 9, pp. 2260-2266, 2009.

[11] I. Gablech, V. Svatoš, O. Caha, M. Hrabovský, J. Prášek, J. Hubálek, et al "Preparation of (001) preferentially oriented titanium thin films by ion-beam sputtering deposition on thermal silicon dioxide", Journal of Materials Science, vol. 51, pp. 3329-3336, 2016.

[12] S. Ashraf, C. S. Blackman, R. G. Palgrave, S. C. Naisbitt and I. P. Parkin, "Aerosol assisted chemical vapour deposition of WO3 thin films from tungsten hexacarbonyl and their gas sensing properties", Journal of Materials Chemistry, vol. 17, pp. 3708-3713, 2007.

[13] N. Singh, PLEASE COMPLETE "Sensing properties of different classes of gases based on the nanowire-electrode junction barrier modulation", Nanoscale, vol. 3, pp. 1760-1765, 2011.

14] T.-Y. Wei, P.-H. Yeh, S.-Y. Lu and Z. L. Wang, "Gigantic Enhancement Sensitivity Using Schottky Contacted Nanowire Nanosensor", Jour. of the American Chemical Society, vol. 131, pp. 17690-17695, 2009.

Received 15 December 2016

Ondřej Chmela received his diploma in microelectronics in 2013 from Brno University of Technology, Faculty of Electrical Engineering and Communication, Czech Republic. $\mathrm{He}$ is currently finishing his $\mathrm{PhD}$ at the same university. His research interest is in the areas of micro/nanostructures development and their fabrication processes. Mainly, he is focused on fabrication of nanowire-based devices for gas-sensing application.

Jakub Sadílek received his diploma in chemistry in 2013 from Brno University of Technology, Faculty of Chemistry. Currently, He is PhD student at CEITEC research institute in Brno, Czech Republic. His current research is focused on fabrication and study of nanostructures and modified surfaces with functional properties applicable for sensors and biosensors.

Stella Vallejos received her $\mathrm{PhD}$ degree from Universitat Rovira i Virgili in 2008. Her current research is generally focused on gas sensing technologies and nanomaterials. She is interested in exploring scalable synthesis methods able to tailor and engineer the sensing properties of nanomaterials, as well as in the development of gas sensing micro/nano systems for applications in safety, security and air quality monitoring.

Jaromír Hubálek received his $\mathrm{PhD}$ degree in 2003 from Brno University of Technology. He works with micro- and nanotechnologies for sensing. He has experiences with gas sensors preparation and electrochemical sensors and instrumentation. He is group leader of Laboratory of Microsensors and nanotechnologies at SIX centre and group of Smart Nanodevices at CEITEC centre. 